\title{
LATTICES OF SUBSEMIVARIETIES OF CERTAIN VARIETIES
}

\author{
AHMAD SHAFAAT
}

(Received 17 March 1969)

To Bernhard Hermann Neumann on his 60th birthday

Communicated by G. B. Preston

For all positive integers $m, n, m \leqq n$, let $\mathscr{V}_{m, n}$ denote the variety of algebras with $m n$-ary operations $\omega_{1}, \cdots, \omega_{m}$ and $n m$-ary operations $\varphi_{1}, \cdots, \varphi_{n}$ satisfying the system of identities:

$$
\begin{aligned}
& \varphi_{i}\left(\omega_{1}\left(x_{1}, \cdots, x_{n}\right), \cdots, \omega_{m}\left(x_{1}, \cdots, x_{n}\right)\right)=x_{i}(i=1, \cdots, n) \\
& \omega_{j}\left(\varphi_{1}\left(x_{1}, \cdots, x_{m}\right), \cdots, \varphi_{n}\left(x_{1}, \cdots, x_{m}\right)\right)=x_{j}(j=1, \cdots, m)
\end{aligned}
$$

The varieties $\mathscr{V}_{m, n}$ are considered by Swierczkowski [1] and by Akataeb and Smirnov [2]. Jonsson and Tarski [3] consider $\mathscr{V}_{m, n}$ in the case $m=1, n=2$. In [2] it is shown that the lattice $L_{m, n}$ of subvarieties of $\mathscr{V}_{m, n}$ is uncountable except when $m=1$ in which case $L_{m, n}$ has a very simple description. In particular, for $n>1$ the lattice $L_{1, n}$ is the two element chain which means that $\mathscr{V}_{1, n}$ has no proper subvarieties.

To consider subvarieties of a variety is a simple general method of classifying its algebras. By considering more general subclasses we can, in general, obtain finer though possibly less simple classifications of algebras of a variety. Semivarieties [4] and quasivarieties seem to provide fairly nice generalizations of the concept of a variety and in the study of many varieties it may be a worthwhile task to consider lattices of their subsemivarieties and subquasivarieties. To take up this task is specially promising in the case of varieties, like $\mathscr{V}_{1, n}$ and the class $\mathscr{G}$ of all abelian groups, that have too few (i.e., less than $2^{\aleph_{0}}$ ) subvarieties ${ }^{1}$.

In this paper we consider the lattices $L_{s}\left(\mathscr{V}_{1, n}\right)$ and $L_{s}(\mathscr{G})$ of subsemivarieties of $\mathscr{V}_{1, n}$ and $\mathscr{G}$ respectively. The lattice $L_{s}\left(\mathscr{V}_{1,1}\right)$ has a rather simple description: $L_{s}\left(\mathscr{V}_{1,1}\right)$ is isomorphic to the lattice $S_{\vee}\left(3^{*}\right)$ of all complete join subsemilattices of $3^{*}$, where $8^{*}$ is obtained from the lattice 8 of positive integers under divisibility by adding a unit $\infty$, say. We show that $L_{s}\left(\mathscr{V}_{1, n}\right)$ has a meet subsemilattice isomorphic to the lattice $S_{\mathrm{v}}, \infty\left(3^{*}\right)$ of join subsemilattices of $3^{*}$ that contain $\infty$.

1 It may be noted that in some cases there may not even be enough subquasivarieties. Thus, for example, the variety of distributive lattices has not only no proper subvarieties but also no proper subquasivarieties. 
The lattice $L_{s}(\mathscr{G})$ is shown to be isomorphic to the lattice $S_{\mathrm{v}, \leq}\left(8^{+} \times 2\right)$ of ideals of $3^{+} \times 2$, where $3^{+}$is obtained from 8 by adding a new zero 0 , say. These results imply that $L_{s}\left(\mathscr{V}_{1, n}\right)$ and $L_{s}(\mathscr{G})$ have cardinality $2^{\aleph_{0}}$ so that subsemivarieties provide reasonably fine classifications of the algebras of these varieties. We also observe that for $n>1$ all subquasivarieties of $\mathscr{V}_{1, n}$ and $\mathscr{G}$ are semivarieties.

We begin by recalling that a class $\mathscr{A}$ of $\Omega$-algebras is called a quasivariety if it is the class of all $\Omega$-algebras satisfying a certain set $\Sigma$ of finite implications, i.e., sentences of the form

$$
\forall x_{1}, \cdots, x_{n}\left(\left(w_{1}=w_{1}^{\prime} \wedge \cdots \wedge w_{m}=w_{m}^{\prime}\right) \rightarrow w=w^{\prime}\right)
$$

where $w, w_{1}, \cdots, w_{m}, w^{\prime}, w_{1}^{\prime}, \cdots, w_{m}^{\prime}$ are $\Omega$-words in $\left\{x_{1}, \cdots, x_{n}\right\} . \mathscr{A}$ is called [4] a semivariety if it is definable by a set of implications of the simpler form

$$
\forall x_{1}, \cdots, x_{n}\left(w_{1}=w_{1}^{\prime} \rightarrow w=w^{\prime}\right) .
$$

In writing implications we shall often omit the quantifiers.

A subquasivariety (subsemivariety) of a quasivariety (semivariety) is a subclass that is also a quasivariety (semivariety).

A join (meet) subsemilattice of a lattice $\langle L ; \vee, \wedge\rangle$ is a subset of $L$ closed under finite joins (meets). A join (meet) subsemilattıce $\langle L ; \vee, \wedge\rangle$ will be called complete if it is closed under infinite joins (meets) whenever they exist in $\langle L ; \vee, \wedge\rangle$.

We first consider $L_{s}\left(\mathscr{V}_{1,1}\right)$.

THEOREM 1. $L_{s}\left(\mathscr{V}_{1,1}\right)$ is isomorphic to the lattice $S_{\mathrm{v},}\left(B^{*}\right)$ of all complete join subsemilattices of $3^{*}$.

Proof: In view of (1) for $m=n=1$ the variety $\mathscr{V}_{1,1}$ consists of algebras $\langle A ; \varphi, \omega\rangle$ where $\varphi, \omega$ are permutations of $A$ and $\omega=\varphi^{-1}$. It follows that within $\mathscr{V}_{1,1}$ every subsemivariety of $\mathscr{V}_{1,1}$ is defined by a set of implications of the form

$$
\varphi^{\mathrm{s}}\left(x_{i}\right)=x_{j} \rightarrow \varphi^{t}\left(x_{l}\right)=x_{k}
$$

where $s, t \in Z^{*}$ and $\varphi^{\infty}(x)=x$ always holds. We prove the simple fact that within $\mathscr{V}_{1,1}$ the sentence (4) is equivalent to an implication of one of the forms:

$$
\begin{aligned}
& \varphi^{s}(x)=x \rightarrow x=y \\
& \varphi^{s}(x)=x \rightarrow \varphi^{t}(x)=x, t \backslash s .
\end{aligned}
$$

If in (4) the variables $x_{i}, x_{j}$ are distinct then (4) is equivalent to the identity $\varphi^{t_{1}}\left(x_{l}\right)=\varphi^{t_{2}}\left(x_{k}\right)$ obtained from $\varphi^{t}\left(x_{l}\right)=x_{k}$ by replacing $x_{j}$ by $\varphi^{s}\left(x_{i}\right)$ throughout. Since $\varphi$ is one-to-one and $\varphi^{\infty}(x)=x$ holds by convention this identity is equivalent to an implication of the form $\varphi^{\infty}(x)=x \rightarrow \varphi^{t}\left(x_{l}\right)=x_{k}$. Thus in (4) we can always take $i=j$. If $l=k$ then $\varphi^{s}(x)=x$ implies $\varphi^{t}(x)=x$ and hence $\varphi^{(s, t)}(x)=x$, where $(s, t)$ is the meet of $s, t$ in $3^{*}$; so that in this case (4) is equivalent to an implication of the form (6). If $l \neq k$ then assume $\varphi^{s}(x)=x$. Then (4) 
implies $\varphi^{t}(x)=y$ for all $y$. Since $\varphi$ and hence $\varphi^{t}$ is a permutation $\forall y \varphi^{t}(x)=y$ is equivalent to saying $\forall y x=y$. Thus in the case $l \neq k$ the implication (4) is equivalent to (5).

We now set up a complete lattice isomorphism $\alpha$ between $L_{s}\left(\mathscr{V}_{1,1}\right)$ and $S_{\vee}\left(3^{*}\right)$. First we define a simple concept. Let $A$ be a set and $\varphi$ a function from $A$ into $A$. By the $\varphi$-order of an element $a$ of $A$ we shall understand the smallest $s \in Z^{*}$ such that $\varphi^{s}(a)=a$. Clearly the $\varphi$-order of every $a \in A$ exists and $\varphi^{s}(a)=a$ if and only if $s$ is divisible by the $\varphi$-order of $a$. Now for every subsemivariety $\mathscr{V}$ of $\mathscr{V}_{1,1}$ we define $\alpha(\mathscr{V})$ to be the set of $s \in Z^{*}$ such that there exists in $\mathscr{V}$ a non-trivial algebra with elements of $\varphi$-order $s$. It is easy to see that $\alpha(\mathscr{V})$ is closed in $3^{*}$ under joins. For let $\left\{s_{r} ; r=1,2, \cdots\right\}$ be a family of integers in $\alpha(\mathscr{V})$ and let $s$ be the join of this family. Let $\mathfrak{A}_{r}, r=1,2, \cdots$, be an algebra in $\mathscr{V}$ having an element $a_{r}$ of $\varphi$-order $s_{r}$. Let $\mathfrak{A}$ be the cartesian product of the $\mathfrak{A}_{r}$. Then the element $a$ of $\mathfrak{A}$ whose $r$ th component is $a_{r}$ has $\varphi$-order $s$. Since semivarieties are closed under the formation of cartesian products, we see that $s \in \alpha(\mathscr{V})$; so that $\alpha$ provides a function from $L_{s}\left(\mathscr{V}_{1,1}\right)$ into $S_{\mathrm{v}}\left(3^{*}\right)$. We first prove that $\alpha$ is onto. Let $Y$ be a subset of $Z^{*}$ closed under joins. For every $s \in Z^{*}$ let $\sigma_{s}$ be the implication $\varphi^{s}(x)=x \rightarrow x=y$ if $Y$ contains no divisors of $s$; otherwise let $\sigma_{s}$ be $\varphi^{s}(x)=$ $x \rightarrow \varphi^{t}(x)=x$, where $t$ is the join of all the divisors of $s$ in $Y$. Let $\mathscr{V}$ be the subsemivariety of $\mathscr{V}_{1,1}$ defined within $\mathscr{V}_{1,1}$ by $\Sigma=\left\{\sigma_{s} ; s \in 3^{*}\right\}$. Then $\alpha(\mathscr{V})=Y$. For if $s \notin Y$ then $\sigma_{s}$ clearly implies that $\mathscr{V}$ cannot contain a non-trivial algebra with elements of $\varphi$-order $s$ and hence $s \notin \alpha(\mathscr{V})$. On the other hand if $s \in Y$, then $\sigma_{s}$ is of the form $\varphi^{s}(x)=x \rightarrow \varphi^{s}(x)=x$ and the cyclic permutation $\varphi=(1,2$, $\cdots, s)$ of order $s$ defines an algebra on $\{1, \cdots, s\}$ in $\mathscr{V}_{1,1}$ satisfying $\Sigma$ which has elements of $\varphi$-order $s$; so that $s \in \alpha(\mathscr{V})$. Next we show that $\alpha$ is one-to-one. Let $\mathscr{V}, \mathscr{V}^{\prime}$ be two subsemivarieties of $\mathscr{V}_{1,1}$ such that $\alpha(\mathscr{V})=\alpha\left(\mathscr{V}^{\prime}\right)$. Let $\sigma$ be an implication of the form (4) that holds in $\mathscr{V}$ but not in $\mathscr{V}^{\prime}$. In case $\sigma$ is equivalent to a sentence of the form $\varphi^{s}(x)=x \rightarrow x=y$, it implies that there are no divisors of $s$ in $\alpha(\mathscr{V})$ and hence also in $\alpha\left(\mathscr{V}^{\prime}\right)$. However since $\sigma$ does not hold in $\mathscr{V}^{\prime}$, there must be a non-trivial algebra in $\mathscr{V}^{\prime}$ in which there exists an element $a$ satisfying $\varphi^{s}(a)=a$. But then the $\varphi$-order of $a$ is a divisor of $s$ that lies in $\alpha\left(\mathscr{V}^{\prime}\right)$, a contradiction. In the other case, when $\sigma$ is equivalent to the form $\varphi^{s}(x)=x \rightarrow$ $\varphi^{t}(x)=x$, a divisor of $s$ which is not a divisor of $t$ cannot lie in $\alpha(\mathscr{V})$ or $\alpha\left(\mathscr{V}^{\prime}\right)$ and we arrive at a contradiction as before. This proves that $\alpha(\mathscr{V})=\alpha\left(\mathscr{V}^{\prime}\right)$ implies $\mathscr{V}=\mathscr{V}^{\prime}$. We conclude the proof by showing that $\mathscr{V} \subseteq \mathscr{V}^{\prime}$ if and only if $\alpha(\mathscr{V}) \subseteq$ $\alpha\left(\mathscr{V}^{\prime}\right)$. The inclusion $\mathscr{V} \subseteq \mathscr{V}^{\prime}$ clearly implies $\alpha(\mathscr{V}) \subseteq \alpha\left(\mathscr{V}^{\prime}\right)$. Let $Y, Y^{\prime} \in S_{\vee}\left(\mathcal{Z}^{*}\right)$ and $Y \subseteq Y^{\prime}$. We described above the set $\Sigma_{Y}=\left\{\sigma_{s} ; s \in B^{*}\right\}$ defining $\alpha^{-1}(Y)$. From this description it is clear that if $\Sigma_{Y^{\prime}}=\left\{\sigma_{s}^{\prime} ; s \in Z^{*}\right\}$ then $\sigma_{s}$ implies $\sigma_{s}^{\prime}$ for all $s \in 3^{*}$ and hence $\alpha^{-1}(Y) \subseteq \alpha^{-1}\left(Y^{\prime}\right)$. This completes the proof of the theorem.

The argument used in the next theorem seems to be of fairly general applicability in obtaining uncountable subsemilattices of lattices of subsemivarieties of semivarieties. 
THEOREM 2. For every positive integer $n$ the lattice $S_{\mathrm{v}, \infty}\left(8^{*}\right)$ of join subsemilattices of $8^{*}$ that contain $\infty$ is embeddable into $L_{s}\left(\mathscr{V}_{t, n}\right)$ as a meet subsemilattice.

Proof: The variety $\mathscr{V}_{l, n}$ is defined in terms of $n$ unary operations $\varphi_{1}, \cdots, \varphi_{n}$ and one $n$-ary operation $\omega$ by the system of identities:

$$
\varphi_{i}\left(\omega\left(x_{1}, \cdots, x_{n}\right)\right)=x_{i}, \omega\left(\varphi_{1}(x), \cdots, \varphi_{n}(x)\right)=x(i=1, \cdots, n) .
$$

Let $\varphi(x)=\omega(x, \cdots, x)$ and let $\mathfrak{M}$ be the lattice of non-trivial subsemivarieties of $\mathscr{V}_{1, n}$ defined within $\mathscr{V}_{1, n}$ by a set of implications of the form (5) or (6). For every $\mathscr{V}$ in $\mathfrak{M}$ we define $\alpha(\mathscr{V})$ as in Theorem 1 . We show that $\alpha(\mathscr{V}) \in S_{\mathrm{v}, \infty}\left(3^{*}\right)$. That $\alpha(\mathscr{V})$ is a join subsemilattice of $8^{*}$ can be proved as in Theorem 1. If $\infty \notin \alpha(\mathscr{V})$ then the join of $\alpha(\mathscr{V})$ is an integer $s$, say, and the identity $\varphi^{s}(x)=x$ holds in $\mathscr{V}$. But this contradicts the fact proved in [2] that $\mathscr{V}_{1, n}$ is equationally complete. Hence $\infty \in \alpha(\mathscr{V})$ for all $\mathscr{V} \in \mathfrak{M}$. Now by one modification of the argument used in the proof of Theorem 1 we can show that $\alpha$ provides an isomorphism between $\mathfrak{M}$ and $S_{v, \infty}\left(3^{*}\right)$. The required modification is the following: In the proof of Theorem 1 we used the algebra $\left\langle\{1, \cdots, s\}, \varphi, \varphi^{-1}\right\rangle \in \mathscr{V}_{1,1}$ in proving that $\alpha$ was onto, where $\varphi$ is the cyclic permutation $(1 \cdots s)$. This time we can use an algebra $\left\langle A ; \varphi_{1}, \cdots, \varphi_{n}, \omega\right\rangle \in \mathscr{V}_{1, n}$ which has elements of $\omega(x, \cdots, x)$-order $s$ and $\infty$ only for some given positive integer $s$. The existence of such an algebra can be proved as follows. Let $A$ be any infinite set. Partition $A$ into sets $A_{s}, A_{\infty}, B$ such that $A_{s}$ has exactly $s$ elements $a_{1}, \cdots, a_{s}$, say, and $A_{\infty}, B, A$ all have the same cardinality. For every $b \in B$ choose a countably infinite subset $A_{\infty}^{b}=\left\{a_{b 1}, \cdots\right.$, $\left.a_{b 2}, \cdots\right\}$ of $A_{\infty}$ such that $\left\{A_{\infty}^{b}\right\}_{b \in B}$ is a partition of $A_{\infty}$. Now define

$$
\begin{array}{ll}
\omega\left(a_{i}, \cdots, a_{i}\right)=a_{i+1}, & i=1, \cdots, s-1 \\
\omega\left(a_{i}, \cdots, a_{i}\right)=a_{1}, & i=s \\
\omega(b, \cdots, b)=a_{b 1}, & b \in B \\
\omega\left(a_{b r}, \cdots, a_{b r}\right)=a_{b r+1}, & b \in B, r=1,2, \cdots
\end{array}
$$

and for other choices of $x_{1}, \cdots, x_{n}$ from $A$ define $\omega\left(x_{1}, \cdots, x_{n}\right)$ such that $\omega$ gives a one-to-one function from $A^{n}$ onto $A$. For every $x \in A$ and $i=1, \cdots, n$ we define $\varphi_{i}(x)$ to be the ith component of the unique $n$-tuple $\left\langle x_{1}, \cdots, x_{n}\right\rangle$ such that $\omega\left(x_{1}\right.$, $\left.\cdots, x_{n}\right)=x$. In this way we get an algebra $\left\langle A ; \varphi_{1}, \cdots, \varphi_{n}, \omega\right\rangle$ which can be easily seen to have the desired property. The proof of the theorem can now be completed by observing that $\mathfrak{M}$ is a meet subsemilattice of $L_{s}\left(\mathscr{V}_{1, n}\right)$. $2^{\mathrm{N}_{0}}$.

COROLlaRY. For every positive integer $n$ the lattice $L_{s}\left(\mathscr{V}_{1, n}\right)$ has cardinality

Proof: In view of Theorem 2 it is sufficient to prove that $S_{v, \infty}\left(3^{*}\right)$ has cardinality $2^{\mathrm{N}_{\mathrm{N}}}$. This follows by noting that every set $P$ of primes determines uniquely the member $\lambda(P)$ of $S_{\mathrm{v}, \infty}\left(8^{*}\right)$ consisting of $\infty$ and all the products of primes in $P$. 
Remark: The variety $\mathscr{V}_{m, n}, m>1, m \leqq n$, was shown in [2] to have uncountably many subvarieties. Combining with Theorem 2 this shows that $L_{s}\left(\mathscr{V}_{m, n}\right)$ has cardinality $2^{\aleph_{0}}$ for all $m, n, m \leqq n$.

Our next easy result gives an interesting property of $\mathscr{V}_{1, n}$.

Remark 3. Every subquasivariety of $\mathscr{V}_{1, n}, n>1$, is a semivariety.

Proof: The identities (7) defining $\mathscr{V}_{1, n}$ imply that

$$
\omega\left(x_{1}, \cdots, x_{n}\right)=\omega\left(x_{1}^{\prime}, \cdots, x_{n}^{\prime}\right) \leftrightarrow x_{1}=x_{1}^{\prime} \wedge \cdots \wedge x_{n}=x_{1}^{\prime} .
$$

From this it is easy to see that if $w\left(x_{1}, \cdots, x_{m}\right)$ is any $\omega$-word such that every one of the variables $x_{1}, \cdots, x_{m}$ actually appears in $w$ then the equivalence

$$
w\left(x_{1}, \cdots, x_{m}\right)=w\left(x_{1}^{\prime}, \cdots, x_{m}^{\prime}\right) \leftrightarrow x_{1}=x_{1}^{\prime} \wedge \cdots \wedge x_{m}=x_{m}^{\prime}
$$

also holds. If $n>1$ we can build an $\omega$-word involving any given number of variaables. In view of this every implication

$$
\left(w_{1}=w_{1}^{\prime} \wedge \cdots \wedge w_{m}=w_{m}^{\prime}\right) \rightarrow w=w^{\prime}
$$

relevant to $\mathscr{V}_{1, n}, n>1$, is equivalent within $\mathscr{V}_{1, n}$ to

$$
w\left(w_{1}, \cdots, w_{m}\right)=w\left(w_{1}^{\prime}, \cdots, w_{m}^{\prime}\right) \rightarrow w=w^{\prime}
$$

where $w$ is an $\omega$-word involving exactly $m$ variables. This proves the theorem.

We now turn to groups.

THEOREM 4. All quasivarieties of abelian groups are semivarieties and their lattice is isomorphic to the lattice $\left.S_{\mathrm{v}}, 3^{+} \times 2\right)$ of ideals of $3^{+} \times 2$.

Proof. Let $\mathscr{G}^{\prime}$ be a class of groups and let $\omega\left(\mathscr{G}^{\prime}\right)$ denote the set of orders of finite cyclic groups in $\mathscr{G}^{\prime}$. Define $s\left(\mathscr{G}^{\prime}\right)=\{0, \infty\}$ or $\{0\}$ according as $\mathscr{G}^{\prime}$ contains the infinite cyclic group or not. Write $\mathrm{O}\left(\mathscr{G}^{\prime}\right)$ for the ordered pair $\left\langle\omega\left(\mathscr{G}^{\prime}\right), s\left(\mathscr{G}^{\prime}\right)\right\rangle$. It is easily seen that $O\left(\mathscr{G}^{\prime}\right)$ is an ideal of $8^{+} \times 2$ if $\mathscr{G}^{\prime}$ is closed under the formation of subgroups and cartesian products. We shall refer to $\mathrm{O}\left(\mathscr{G}^{\prime}\right)$ as the order of $\mathscr{G}^{\prime}$.

We need to recall two well-known facts. Firstly, every abelian group is the union of a countable ascending chain of direct products of cyclic groups. Secondly, (and this is easy to verify) quasivarieties are closed under taking unions of countable ascending chains. These two facts together immediately show that $\left({ }^{*}\right)$ Every quasivariety of abelian groups consists of all the unions of countable ascending chains of direct products of its cyclic groups and is therefore determined completely by its order.

Now let $\Sigma\left(\mathscr{G}^{\prime}\right)$ be the set of implications $\sigma_{n}: x^{n}=1 \rightarrow x^{\theta(n)}=1$, where $n \in \omega\left(\mathscr{G}^{\prime}\right) \cup s\left(\mathscr{G}^{\prime}\right)$ and $\theta(n)$ is the largest member of $\omega\left(\mathscr{G}^{\prime}\right) \cup s\left(\mathscr{G}^{\prime}\right)$ not exceeding $n$. It is easy to see (Cf. proof of Theorem 1 ) that the semivariety $\mathscr{G}^{\prime \prime}$ defined by $\Sigma\left(\mathscr{G}^{\prime}\right)$ is such that $\mathrm{O}\left(\mathscr{G}^{\prime}\right)=\mathrm{O}\left(\mathscr{G}^{\prime \prime}\right)$. If $\mathscr{G}^{\prime}$ is a quasivariety this implies, in view of $\left({ }^{*}\right)$, that $\mathscr{G}^{\prime}=\mathscr{G}^{\prime \prime}$ and the theorem is proved. 
Theorem 4 has the following simple generalization.

THEOREM 5. For every quasivariety $\mathscr{G}^{\prime}$ of groups the lattice $L_{s}\left(\mathscr{G}^{\prime}\right)$ has a sublattice isomorphic to the lattice of ideals of the order $\mathrm{O}\left(\mathscr{G}^{\prime}\right)$ of $\mathscr{G}^{\prime}$.

Theorem 5 follows by noting that the quasivariety $\mathscr{G}^{\prime \prime}$ of abelian groups in $\mathscr{G}^{\prime}$ is such that $\mathrm{O}\left(\mathscr{G}^{\prime \prime}\right)=\mathrm{O}\left(\mathscr{G}^{\prime}\right)$ and that $L_{s}\left(\mathscr{G}^{\prime \prime}\right)$ is a sublattice of $L_{s}\left(\mathscr{G}^{\prime}\right)$, and then proceeding as in the proof of Theorem 4.

\section{References}

[1] S. Swierczkowski, 'On isomorphic free algebras', Fund. Math. 50 (1961), 35-44.

[2] A. A. Akataeb and D. M. Smirnov, 'Lattices of subvarieties of varieties of algebras' [Russian] Algebra i Logica 7 (1968), 5-25.

[3] Bjarni Jonsson and Alfred Tarski, 'On two properties of free algebras', Math. Scand. 9 (1961), 95-101.

[4] Ahmad Shafaat, 'Characterizations of some universal classes of algebras', J. London Math. Soc. 2 (1970), $385-388$.

University of Manitoba

Winnipeg, Manitoba

Canada 